Received Date : 15-May-2016

Revised Date : 20-Oct-2016

Accepted Date : 24-Oct-2016

Article type : Original Article

\title{
Factors affecting SPF in vitro measurement and correlation with in vivo results
}

Aleksandra Dimitrovska Cvetkovska, ${ }^{2}$ Stefano Manfredini, ${ }^{1,2,3}$ Paola Ziosi, ${ }^{3}$ Sonia Molesini, ${ }^{3}$ Valeria Dissette, ${ }^{2}$ Ilenia Magri, ${ }^{3}$ Chiara Scapoli, ${ }^{2}$ Alberto Carrieri, ${ }^{2}$ Elisa Durini, ${ }^{1,2}$ Silvia Vertuani ${ }^{1,2,3}$

${ }^{1}$ School of Pharmacy, Master Course in Cosmetic Science and Technology, ${ }^{2}$ Department of Life Sciences and Biotechnology, University of Ferrara, via L. Borsari 46, 44121 Ferrara, Italy ${ }^{3}$ Ambrosialab srl, via Mortara 171, 44121 Ferrara, Italy

\section{Corresponding author Mail id: mv9@unife.it}

\section{Abstract}

OBJECTIVE: The in vitro evaluation of SPF is still a problem due to the lack of repeatability and correlation between the in vitro and in vivo data and many authors are currently working to develop an internationally harmonized method.(1) Very recently, the use of several "adjuvant" ingredients such as boosters, antioxidants, immuno-modulators, solvents and film forming ingredients have further complicated the pattern for product developers, that should frequently run in vivo test. The aim of this study is to understand if a simple and cheap in vitro method could be optimized in order to provide both statistically repeatable and predictive SPF measurement.

METHODS: In vitro SPF assessments were carried out on 75 commercial products. The SPF was measured according to two laboratory methods ( $A$ and $B$ ), using different substrates (PMMA and surgical tape Transpore ${ }^{\mathrm{TM}}$ ), quantity of product, spectrophotometers. In order to evaluate if a standard technique of spreading could lead to a statistically reliable result, we applied different spreading pressure (100 g and $200 \mathrm{~g})$. Furthermore, we investigate if other parameters characterizing the product (SPF Category, Filter and Texture) might represent statically significant variables affecting the measures. We then compared the results obtained from in vitro SPF measure of 11 products to in vivo SPF, in order to assess the predictivity of in vitro methods.

This article has been accepted for publication and undergone full peer review but has not been through the copyediting, typesetting, pagination and proofreading process, which may lead to differences between this version and the Version of Record. Please cite this article as doi: $10.1111 /$ ics. 12377

This article is protected by copyright. All rights reserved. 
RESULTS: Several problems were encountered in confirming the weakness of the in vitro procedures. Pressure, SPF Category, Filter and Texture did not affect significantly the results. Overall best results were obtained with the B2 method that in terms of repeatability and predictivity provided statistically better results. Method A with Transpore ${ }^{\mathrm{TM}}$ tape showed better in vitro-in vivo correlation than Method B with PMMA plates.

CONCLUSION: In our investigation we demonstrated that it is possible for a single laboratory to optimize internal methods and protocols to achieve repeatable and predictive in vitro results, but it is extremely difficult to develop methods reproducible and equally reliable in different laboratories, probably due to "external variables" (eg. environmental, operator), which are difficult to control.

Key words: Claim substantiation, Formulation, Spectroscopy, SPF in vitro, Sunscreens

\section{Introduction}

Over and incorrect exposure to sunlight has harmful consequences on human skin. Oxidative effects especially due to UV rays are the main cause of aged skin, sunburn and skin cancer. Therefore, sunscreens have become widely used for the prevention of short and long-term skin damage and for this reason consumers are offered a wide range of cosmetic products with protection against UV irradiation.

The sun protection factor (SPF) labelled on sunscreen products determines the amount of protection against the erythema induced by UV radiation. This value is widely recognized by the general public as a measure for the protection offered by a sunscreen preparation against sunburn and the classification of the SPF has to be proved by the manufacturers.

In the European Union sunbathing products are listed as cosmetics: their management must follow the articles of the Regulation EC 1223/2009 of the European Parliament on Cosmetic Products (2). Moreover, regarding the efficacy evaluation, the relevant guideline is the Commission Recommendation 2006/647 on the efficacy of sunscreen products and the claims made relating thereto (3). In this document a particular notice should be placed at the Preliminary Considerations, especially at points 16) and 17) where it clarifies that, in order to ensure reproducibility and comparability of the recommended minimum protection against UVB and UVA radiations, the methods used to evaluate the products must be specific, officially and internationally recognized. At the time of the Commission Recommendation 2006/647 draft, the main reference methods were International Sun Protection Factor Test Method for SPF calculation and the Persistent Pigment

This article is protected by copyright. All rights reserved. 
Darkening Method for UVAPF, both based on in vivo techniques. Furthermore the Recommendation states that preference should be given to in vitro testing methods delivering equivalent results, as in vivo methods raise ethical concerns.

According to this, industry increased efforts to develop alternative methods and in 2012 a new method for in vitro UVA protection assessment was established and standardized in the ISO 24443:2012(4), whereas for the SPF determination the ISO 24444:2010(5) is still the reference method, despite providing an in vivo procedure carried out on human volunteers.

Investigation through the literature in this field highlights that the main concern, about the in vitro techniques used for SPF evaluation, is the lack of data to support method reproducibility and correlation to in vivo results. $(6,7)$ The in vivo measurement remains therefore the "gold standard" and product developers should perform the in vivo test during all the phases of the development, not just on the final product, which obviously bring back the attention on costs and the ethical issue.

At the present there are several in vitro methods, all used just for screening or developing purposes (8). The first method was proposed by Diffey in 1989 and is still the most accredited reference (9). A sunscreen in vitro test is based on spectrophotometric measurement, as absorbance (calculated from trasmittance) or reflectance (10), of a thin film of product applied on suitable UV transparent substrates. The differences in all the methods are basically in the type of plastic support used and the way to apply the sunscreen on it $(11,12)$. Starting from Diffey's procedure, the supports used can range from plastic perforated surgical tape, as Transpore ${ }^{\mathrm{TM}}$ tape, to standardized plastic plates, as Polymethymethacrilate (PMMA) plates, with the amount of product applied varying from 0.7 to 2.0 $\mathrm{mg} / \mathrm{cm}^{2}$. An accurate literature search highlighted that the procedure followed to apply and spread the sunscreen on the plate is a very critical phase of the measure, where the error is increased the most (13). Several factors and variables affect the accuracy and the repeatability of the spectrophotometric measures, such as the different composition of filters (14), the formulation of the sunscreens (15), the thickness and the homogeneity of sunscreen applied (16), the type of spectrophotometer (17), the substrates and their relative roughness (18).

In this study, we first evaluated if a standard technique of spreading could lead to a statistically reliable result, focusing the efforts on controlling spreading pressure. Spreading pressure is sure enough a variable capable to influence consistently the results: according to the pressure exerted, the film thickness obtained on the substrate can vary, it was demonstrated in fact that an increased application pressure reduces the in vitro SPF (19).

This article is protected by copyright. All rights reserved. 
Hence, in order to systematically evaluate the influence of spreading pressure on the result, but mostly to understand if the SPF calculated could be predictive of the in vivo measurement - the gold standard for regulatory purposes - we performed comparative assessment, using two different in vitro methods and adopting different substrates (Transpore ${ }^{\mathrm{TM}}$ Tape and sand blasted PMMA plates). Each method was performed twice on the same product, first using a 100g spreading pressure and later 200g spreading pressure.

The use of Transpore ${ }^{\mathrm{TM}}$ Tape, in determining in vitro SPF may be seem obsolete, standing the availability of more standardisable substrates (e.g. PMMA plates). However, during our studies we observed that, more often than supposed, Transpore ${ }^{\mathrm{TM}}$ Tape is still used for preliminary screening purposes due to its cheap nature.

We thus included also a method based on Transpore ${ }^{\mathrm{TM}}$ Tape, in order to assess whether, by controlling application pressure, it could be possible to improve its performance.

Therefore, the methods proposed were carried out at two different pressures, of $100 \mathrm{~g}$ and $200 \mathrm{~g}$, on two different substrates, Transpore ${ }^{\mathrm{TM}}$ Tape and sand blasted PMMA plates.

We analyzed 75 commercial sunscreen products, with different SPF label, different types of filter and different formulations. Both the spreading techniques were tested using two different instruments; we then compared the results obtained from in vitro SPF measure of 11 products with in vivo SPF. An in vitro SPF method can be considered adequate and predictive only if the results of the analysis correlate to values obtained from in vivo method.

\section{Materials and Methods}

\section{Substrates}

In vitro approaches consist in applying a thin film of sunscreen product on an artificial substrate and test, via spectrophotometric measures, the amount of UV radiation passing through the film.

Several different artificial substrates are available for this type of analysis; the substrate should be as close as possible to the physical characteristics of the skin.

Among the substrates available for this purpose, the two substrates used in this study to analyze the sunscreen products were Transpore ${ }^{\mathrm{TM}}$ tape and PMMA plates.

This article is protected by copyright. All rights reserved. 
- Transpore ${ }^{\mathrm{TM}}$ tape: it is a surgical tape, provided by 3M Company Health Care (Maine, USA). It is used according to the Diffey-Robson method; this tape has a perforated structure and it allows distributing the sunscreen sample in a way similar to the irregular surface of the skin.

- Sand blasted PMMA plates: this substrate is easily handled and can be supplied with a reproducible roughness. WW5 PMMA plates have been purchased from Schonberg GmbH (Munich, Germany). The plates, used in this study, have an area of $25 \mathrm{~cm}^{2}$ and standardized $5 \mu \mathrm{m}$ roughness. The features of this substrate meet the recommendation of ISO 24443 for in vitro UVA protection assessment.

\section{Sunscreens}

\section{Commercial products}

All the products were gifted to or purchased by our laboratory from public pharmacies in EU, Canada, US, and Australia. We decided to include products with different SPF labeled values, from 6 to 50+, with different type of filters and texture. In Table I, the list of tested products is reported; for each product the SPF labelled value is shown together with the type of filter (chemical, C, or physical, P or both, CP) and the texture, according to the viscosity (from "liquid" to "fluid", "creamy" and "paste"). In the second part of the experiment we performed in vivo SPF measurement just on 11 out of the 75 products: the decision followed the purpose to test on human volunteers just formulations of which the exact composition of filters was available to us.

Table I: List of products tested and characteristics.

$\begin{array}{ccccc}\text { Product } & \text { SPF } & \text { Labelled } & \text { Filter } & \text { Texture } \\ & \text { Category } & \text { SPF } & & \\ 1 & \text { Low } & 6 & \text { C } & \text { Liquid } \\ 2 & \text { Low } & 6 & \text { CP } & \text { Fluid } \\ 3 & \text { Low } & 10 & \text { P } & \text { Paste } \\ 4 & \text { Low } & 10 & \text { C } & \text { Fluid } \\ 5 & \text { Low } & 10 & \text { C } & \text { Fluid } \\ 6 & \text { Low } & 10 & \text { C } & \text { Fluid } \\ 7 & \text { Low } & 10 & \text { C } & \text { Liquid } \\ 8 & \text { Medium } & 15 & \text { C } & \text { Fluid } \\ 9 & \text { Medium } & 15 & \text { CP } & \text { Liquid } \\ 10 & \text { Medium } & 15 & \text { C } & \text { Fluid } \\ 11 & \text { Medium } & 15 & \text { CP } & \text { Fluid }\end{array}$

This article is protected by copyright. All rights reserved. 


\begin{tabular}{|c|c|c|c|c|}
\hline 12 & Medium & 15 & $\mathrm{C}$ & Creamy \\
\hline 13 & Medium & 15 & $C$ & Fluid \\
\hline 14 & Medium & 15 & $\mathrm{C}$ & Fluid \\
\hline 15 & Medium & 15 & $\mathrm{CP}$ & Fluid \\
\hline 16 & Medium & 15 & $\mathrm{C}$ & Liquid \\
\hline 17 & Medium & 15 & $\mathrm{P}$ & Fluid \\
\hline 18 & Medium & 20 & $P$ & Paste \\
\hline 19 & Medium & 20 & C & Creamy \\
\hline 20 & Medium & 20 & $\mathrm{CP}$ & Fluid \\
\hline 21 & Medium & 20 & $\mathrm{C}$ & Creamy \\
\hline 22 & Medium & 20 & $P$ & Fluid \\
\hline 23 & Medium & 20 & $\mathrm{P}$ & Creamy \\
\hline 24 & Medium & 20 & $\mathrm{CP}$ & Fluid \\
\hline 25 & High & 30 & $\mathrm{CP}$ & Creamy \\
\hline 26 & High & 30 & $\mathrm{CP}$ & Creamy \\
\hline 27 & High & 30 & $\mathrm{CP}$ & Creamy \\
\hline 28 & High & 30 & $\mathrm{CP}$ & Fluid \\
\hline 29 & High & 30 & C & Fluid \\
\hline 30 & High & 30 & $P$ & Paste \\
\hline 31 & High & 30 & $P$ & Paste \\
\hline 32 & High & 30 & $\mathrm{CP}$ & Fluid \\
\hline 33 & High & 30 & C & Creamy \\
\hline 34 & High & 30 & C & Fluid \\
\hline 35 & High & 30 & $\mathrm{CP}$ & Liquid \\
\hline 36 & High & 30 & $\mathrm{CP}$ & Fluid \\
\hline 37 & High & 30 & C & Liquid \\
\hline 38 & High & 30 & $\mathrm{CP}$ & Fluid \\
\hline 39 & High & 30 & $\mathrm{CP}$ & Paste \\
\hline 40 & High & 30 & $\mathrm{CP}$ & Paste \\
\hline 41 & High & 30 & C & Fluid \\
\hline 42 & High & 30 & C & Fluid \\
\hline 43 & High & 40 & $\mathrm{CP}$ & Fluid \\
\hline 44 & High & 50 & $\mathrm{CP}$ & Fluid \\
\hline 45 & High & 50 & P & Paste \\
\hline
\end{tabular}

This article is protected by copyright. All rights reserved. 


\begin{tabular}{|c|c|c|c|c|}
\hline 46 & High & 50 & C & Creamy \\
\hline 47 & High & 50 & $\mathrm{CP}$ & Creamy \\
\hline 48 & High & 50 & $P$ & Fluid \\
\hline 49 & High & 50 & $\mathrm{CP}$ & Fluid \\
\hline 50 & High & 50 & $\mathrm{CP}$ & Paste \\
\hline 51 & High & 50 & $\mathrm{CP}$ & Fluid \\
\hline 52 & High & 50 & $\mathrm{CP}$ & Fluid \\
\hline 53 & High & 50 & $\mathrm{CP}$ & Fluid \\
\hline 54 & Very High & 60 & $\mathrm{CP}$ & Fluid \\
\hline 55 & Very High & $100+$ & $\mathrm{CP}$ & Liquid \\
\hline 56 & Very High & $50+$ & C & Liquid \\
\hline 57 & Very High & $50+$ & $\mathrm{CP}$ & Creamy \\
\hline 58 & Very High & $50+$ & $\mathrm{CP}$ & Fluid \\
\hline 59 & Very High & $50+$ & $\mathrm{CP}$ & Paste \\
\hline 60 & Very High & $50+$ & $\mathrm{CP}$ & Liquid \\
\hline 61 & Very High & $50+$ & $\mathrm{CP}$ & Creamy \\
\hline 62 & Very High & $50+$ & $\mathrm{CP}$ & Creamy \\
\hline 63 & Very High & $50+$ & $\mathrm{CP}$ & Fluid \\
\hline 64 & Very High & $50+$ & $\mathrm{CP}$ & Creamy \\
\hline 65 & Very High & $50+$ & $\mathrm{CP}$ & Fluid \\
\hline 66 & Very High & $50+$ & $C P$ & Creamy \\
\hline 67 & Very High & $50+$ & $C P$ & Creamy \\
\hline 68 & Very High & $50+$ & C & Creamy \\
\hline 69 & Very High & $50+$ & $\mathrm{CP}$ & Fluid \\
\hline 70 & Very High & $50+$ & $C P$ & Fluid \\
\hline 71 & Very High & $50+$ & C & Fluid \\
\hline 72 & Very High & $50+$ & $\mathrm{CP}$ & Fluid \\
\hline 73 & Very High & $50+$ & $\mathrm{CP}$ & Creamy \\
\hline 74 & Very High & $50+$ & $\mathrm{CP}$ & Fluid \\
\hline 75 & Very High & $50+$ & $C P$ & Liquid \\
\hline
\end{tabular}

This article is protected by copyright. All rights reserved. 


\section{Spectrophotometric measurements}

Transmittance and absorbance measurements were carried out with two different spectrophotometers in respect to the two different substrates.

With PMMA plates, the measurements were carried out by a SHIMAZHU UV-2600 provided of integrating sphere ISR $260060 \mathrm{~mm}$ and coupled with a SPF determination software and a PMMA plate with approximately $15 \mu \mathrm{l}$ of glycerin served as reference. With Transpore ${ }^{\mathrm{TM}}$ Tape, the spectrophotometer was a Jasco V530 coupled with a Jasco SPF determination software and an untreated piece of tape used as reference. The choice to use two different instruments follows the idea to compare a traditional low expensive method to a more recent and expensive one. In both methods, the spectra were recorded from $290 \mathrm{~nm}$ up to $400 \mathrm{~nm}$ with a wavelength increment step set at $1 \mathrm{~nm}$.

\section{In vitro method based on spectral measurements}

The products were applied on the two selected substrates with two different pressures, monitored by a scale; all the other conditions were kept identical, such as the operator, the quantity of the product applied according to the substrate and the room temperature. None of the samples were pre-irradiated because testing the photostability was not the aim of the present study.

\section{Method A - Based On Diffey-Robson's Method}

The support used is a Transpore ${ }^{\mathrm{TM}}$ surgical perforated tape, cut to have an area of $20 \mathrm{~cm}^{2}$, in which an amount of $0,0400 \mathrm{~g} \pm 0,002 \mathrm{~g}\left(2 \mathrm{mg} / \mathrm{cm}^{2}\right)$ of product is weighed and laid in small spots through all the area. The tape is then positioned on a scale where the spreading phase is carried out with a finger cot and performing a pattern of 6 movements in horizontal, vertical and circular directions, checking the pressure applied in all the movements. For all the products the spreading pressure is first of $100 \mathrm{~g}$ (Method A1) and in the second set of tests of $200 \mathrm{~g}$ (Method A2). Three tapes were prepared for each product, recording 5 measures each, collecting therefore 15 spectra.

\section{Method B - Based on ISO 24443 procedure}

This method incorporates many of the recommendations issued by the ISO 24443:2012 standard for the in vitro determination of UVA protection.

The support used is a PMMA (poly methyl methacrylate) plastic plate with an area of $25 \mathrm{~cm}^{2}$ and standardized $5 \mu \mathrm{m}$ roughness, in which an amount of $0.0320 \mathrm{~g} \pm 0,0005 \mathrm{~g}\left(1,3 \mathrm{mg} / \mathrm{cm}^{2}\right)$ of product is weighed and laid in small spots through all the area. The plate is then positioned on a scale where the spreading phase is carried out performing with pre-saturated finger cot a sequence of 6

This article is protected by copyright. All rights reserved. 
movements in horizontal, vertical and circular direction, checking the pressure applied throughout the spreading.(21) For all the products the spreading pressure is first of $100 \mathrm{~g} \pm 15 \mathrm{~g}$ (Method B1) and in a second analysis of $200 \mathrm{~g} \pm 15 \mathrm{~g}$ (Method B2). Before the measurement the sample lies for a minimum of 15 minutes in a dark place, allowing the evaporation of volatile components. Three plates were prepared for each product, recording 5 measures each, collecting therefore 15 spectra.

\section{Calibration of the In vitro SPF}

The calibration of the operator and/or the device is controlled by the use of a reference sunscreen formulation with SPF 16.(22) The SPF test results for the reference has to lie between 14 and 18, otherwise the test has to be considered invalid and should be repeated.

The test is performed in triplicate for both the substrates, PMMA plate and Transpore ${ }^{\mathrm{TM}}$ Tape, and for both the methods, $100 \mathrm{~g}$ and $200 \mathrm{~g}$ of pressure.

\section{In vitro SPF calculation}

The following definition was used for the in vitro Sun Protection Factor (SPF) calculated from the spectral absorbance characteristics described above(23):

$$
\text { In vitro } S P F=\frac{\int_{\lambda=290 \mathrm{~nm}}^{\lambda=400 \mathrm{~nm}} E(\lambda) I(\lambda) d(\lambda)}{\int_{\lambda=290 \mathrm{~nm}}^{\lambda=400 \mathrm{~nm}} E(\lambda) I(\lambda) 10^{-A(\lambda)} d(\lambda)}
$$

where:

$E(\lambda)=$ erythema action spectrum (CIE-1987) at wavelength $\lambda$.

$I(\lambda)=$ spectral irradiance received from the UV source at wavelength $\lambda$.

$A(\lambda)=$ monochromatic absorbance of the test product layer at wavelength $\lambda$

$\mathrm{d}(\lambda)=$ wavelength step $(1 \mathrm{~nm})$.

The in vitro SPF could be calculated through sunscreens UV Transmittance, $T(\lambda)$, applying the same equation with $A(\lambda)=-\log [T(\lambda)]$.

\section{In vivo evaluation of products}

The in vivo method according to ISO 24444:2010 standard and European Recommendation 647/2006 was applied to determine the SPF value for 11 selected sunscreen products. As previously stated, for ethical reasons only products available with their exact composition were tested on human volunteers. In this study, 10 subjects per product, female and male, with age ranging from 20

This article is protected by copyright. All rights reserved. 
to 35 years, were tested. Their skin photo type was chosen with sun sensitivity categories of type I, II and III according to Fitzpatrick. All volunteers had been informed in detail before signing a written declaration of consent.

A skin area on the back, $35 \mathrm{~cm}^{2}$ was irradiated with different UV irradiation doses, so that the unprotected minimal erythema dose (MEDu) was determined.

For in vivo SPF determination, a Multiport UV Solar Simulator Model 601, 150 W, was used,-which emits ultraviolet radiation in the region between 290 and $400 \mathrm{~nm}$ from 6 independent outputs. Each output is adjusted on scalar UV doses, set according to parameters tabulated by the instrument.

All sunscreen products were applied in a thin film of $2,00 \pm 0,05 \mathrm{mg} / \mathrm{cm}^{2}$ in the selected area on the back. The product distribution was reached by a gentle massage using a finger cot, at least 15 minutes before the irradiation started. $20 \pm 4$ hours after the UV exposure, MEDp was determined. The SPF was then calculated as described in the ISO 24444:2010 standard.

$$
S P F_{i}=\frac{M E D_{\text {protected skin }}}{M E D_{\text {unprotcted skin }}}=\frac{M E D_{p}}{M E D_{u}}
$$

The SPF result for the test product and for the reference sunscreen formulation is calculated as the arithmetical mean of all valid individual SPF values.

\section{Criteria of interpretation}

The evaluation of the repeatability of the data was carried out taking as reference the ISO Standard 24443 parameters, where the document states that, starting from "s", the following calculations is to be done:

$$
c=\frac{t \times s}{\sqrt{n}}
$$

so that $C l 95 \%=S P F+c$ to $S P F-c$.

$$
C I[\%]=\frac{100 \times c}{S P F}
$$

Where $c$ is the confidence interval (95\% confidence level), $s$ is best estimate of population standard deviation, $t$ is the T statistic and $n$ is the number of measures.

The ISO standard established that the obtained $\mathrm{Cl}$ [\%] value should not be greater than $17 \%$, otherwise the result should be considered not statistically significant. The same criteria of 
interpretation are listed also for in vivo SPF in the ISO standard 24444 and were therefore applied for in vivo data collected in this study.

With the final aim to comparatively evaluate the method, in terms of repeatability, we considered the mean $\mathrm{Cl} \%$ for all the valid data $(\mathrm{Cl} \%<17 \%)$ for each method and the values obtained were statistically compared by a two-way analysis of variance (ANOVA), taking Method and Pressure as variables.

Later we evaluated if the other parameters characterizing the product (Category, Filter and Texture) could represent statically significant variables in comparison to Method and Pressure.

\section{Results and Discussion}

In the following table (Table II) are listed all the results obtained from the experiment. The columns in vitro SPF, SD and Cl\% obtained from the spectrophotometric measurements. The final row reports the mean $\mathrm{Cl} \%$ calculated on the valid samples. As above explained we calculated the $\mathrm{Cl} \%$ to exclude analyses that were not repeatable. The reported in vitro SPF is the mean of the values measured. The results are summarized in Table III.

Prod.

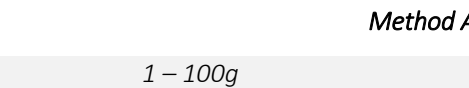

in vitro SPF SD

$9,63 \quad 2,445$

$11,71 \quad 1,619$

$8,74 \quad 1,175$

19,79

$15,44 \quad 2,587$

26,10

23,88

19,12

19,50

23,05

29,80

4,99

25,28

21,08

22,88

$\begin{array}{ll}22,88 & 1,505 \\ 39,91 & 5,362\end{array}$

5,362

0,258

0,437

28,94

38,16

$7,957 \quad 10,37 \%$
2,995

thod A

$\mathrm{Cl} \%$ in vitro SPF $\quad$ SD

\section{$12,63 \%$}

$6,88 \%$

$6,68 \%$

$11,64 \%$

$8,33 \%$

$2,81 \%$

$9,96 \%$

$11,78 \%$

0,475

$1,21 \%$

$9,40 \%$

$6,26 \%$

1,246

4,308

$12,43 \%$

$8,48 \%$

$3,86 \%$

$3,27 \%$

$6,68 \%$

$4,51 \%$

4,31\%

5,15\%
12,79

13,52

8,56

25,02

20,27

23,07

28,00

29,73

25,30

26,84

28,43

8,77

19,99

18,99

25,88

35,10

3,82

5,67

34,37

42,74
SD

2,715

1,856

2,516

4,344

5,457

2,088

3,980

4,193

4,766

7,985

7,894

2,551

3,716

5,251

2,788

9,678

0,476

1,242

5,782

5,896

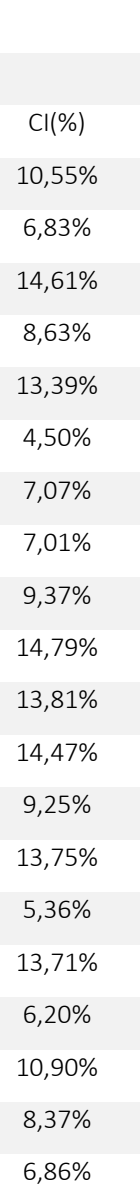

Method B
$1-100 g$

in vitro SPF SD

$6,58 \quad 1,07$

9,28

5,53

11,40

14,18

11,33

14,36

23,36

15,58

12,43

14,11

6,46

12,23

16,73

32,50

28,59

3,92

3,65

25,84

38,68
0,38

0,61

0,89

0,97

0,56

3,77

2,80

0,91

1,49

0,82

0,34

2,08

1,86

3,29

1,70

0,35

0,57

$2,94 \quad 6,30 \%$

$2,88 \%$

$9,44 \%$

$6,15 \%$

$5,60 \%$

$3,29 \%$

$4,91 \%$

$8,58 \%$
$5,90 \quad 8,44 \%$

$8,98 \%$

$6,30 \%$ 2-200g

$\mathrm{Cl}(\%) \quad$ in vitro SPF SD

5,19

0,96

0,35

1,03

0,43

0,75

1,08

3,19

1,00

0,38

4,29

$0,21-1,98 \%$
$\mathrm{Cl}(\%)$

$10,30 \%$

$2,20 \%$

$8,53 \%$

$2,00 \%$

$3,68 \%$

$5,11 \%$

$11,68 \%$

$4,69 \%$

$3,43 \%$

$1,53 \%$

$11,08 \%$

1,98\%

$9,07 \%$

$4,88 \%$

$3,24 \%$

5,53\%

3,98\%

9,23\%

$7,37 \%$

$4,10 \%$

This article is protected by copyright. All rights reserved. 







\begin{tabular}{|c|c|c|c|c|c|c|c|c|c|c|c|c|}
\hline 67 & 66,97 & 9,864 & $7,32 \%$ & 67,15 & 0,895 & $0,66 \%$ & 50,46 & 5,70 & $6,26 \%$ & 60,88 & 7,29 & $6,64 \%$ \\
\hline 68 & 45,62 & 19,560 & $21,32 \% *$ & 52,51 & 6,901 & $6,54 \%$ & 67,43 & 6,12 & $5,03 \%$ & 18,29 & 1,16 & $3,51 \%$ \\
\hline 69 & 26,78 & 4,967 & $9,22 \%$ & 26,25 & 6,871 & $13,02 \%$ & 59,58 & 4,00 & $3,72 \%$ & 65,52 & 4,98 & $4,21 \%$ \\
\hline 70 & 25,27 & 4,599 & $9,05 \%$ & 45,54 & 2,488 & $2,72 \%$ & 44,30 & 7,46 & $9,33 \%$ & 53,38 & 2,02 & $2,09 \%$ \\
\hline 71 & 40,02 & 3,272 & $4,07 \%$ & 60,04 & 7,000 & $5,80 \%$ & 45,81 & 3,95 & $4,77 \%$ & 58,30 & 2,51 & $2,38 \%$ \\
\hline 72 & 28,34 & 4,839 & $8,49 \%$ & 29,27 & 7,423 & $12,61 \%$ & 43,71 & 12,75 & $16,15 \%$ & 42,16 & 13,11 & $17,22 \% *$ \\
\hline 73 & 29,02 & 4,124 & $7,07 \%$ & 29,05 & 2,807 & $4,81 \%$ & 20,50 & 5,60 & $15,14 \%$ & 34,45 & 1,21 & $1,95 \%$ \\
\hline 74 & 70,74 & 9,566 & $6,73 \%$ & 49,69 & 5,609 & $5,61 \%$ & 65,05 & 1,92 & $1,63 \%$ & 61,94 & 4,34 & $3,88 \%$ \\
\hline 75 & 39,13 & 8,482 & $10,78 \%$ & 42,98 & 7,763 & $8,98 \%$ & 17,21 & 0,90 & $2,91 \%$ & 28,94 & 7,57 & $14,48 \%$ \\
\hline \multicolumn{3}{|c|}{ Mean $\mathrm{Cl} \%$ of valid samples } & $8,01 \%$ & & & $7,79 \%$ & & & $6,27 \%$ & & & $5,64 \%$ \\
\hline
\end{tabular}

Table II: analytical and statistical results. ${ }^{*}$ indicates invalid $\mathrm{Cl} \%$ values.

\begin{tabular}{|c|c|c|c|c|}
\hline & \multicolumn{4}{|c|}{ Method } \\
\hline & A1 & A2 & B1 & B2 \\
\hline Not Valid & 4 & 1 & 2 & 2 \\
\hline Valid & 71 & 74 & 73 & 73 \\
\hline Mean Cl\% & $8.01 \%$ & $7.79 \%$ & $6,67 \%$ & $5.64 \%$ \\
\hline
\end{tabular}

Table III. Summary of results.

Starting from the statistical results presented in Table IV, we were able to highlight that just the method exerts some influence on the results, being statistically significant $(p<0.00001)$. Method $B$ showed mean $\mathrm{Cl} \%$ values lower than Method A (Figure 1) confirming, as expected, that the use of PMMA plates give more reproducible results than the tape, regardless of the pressure applied to spread the product. The pressure indeed does not influence the analysis in a statistically significant way $(p>0,05)$ and comparing different application pressures on the same substrate, no statistically significant difference subsists in terms of repeatability.

\begin{tabular}{|c|c|c|c|c|c|}
\hline & SS & df & MS & F & p \\
\hline Intercept & 13954.89 & 1 & 13954.89 & 1086.439 & 0.000000 \\
\hline Method & 276.41 & 1 & 276.41 & 21.520 & $\mathbf{0 . 0 0 0 0 0 5}$ \\
\hline Pressure & 13.28 & 1 & 13.28 & 1.034 & $\mathbf{0 . 3 1 0 0 3 7}$ \\
\hline Method*Pressure & 3.00 & 1 & 3.00 & 0.234 & $\mathbf{0 . 6 2 9 1 1 8}$ \\
\hline Error & 3686.40 & 287 & 12.84 & & \\
\hline
\end{tabular}

Table IV. Statistical analysis Mean $\mathrm{Cl} \%$ vs Method and Pressure: the mean $\mathrm{Cl} \%$ values were statistically compared by a two-way analysis of variance (ANOVA), taking Method and Pressure applied as variables.

This article is protected by copyright. All rights reserved. 
Method Comparison

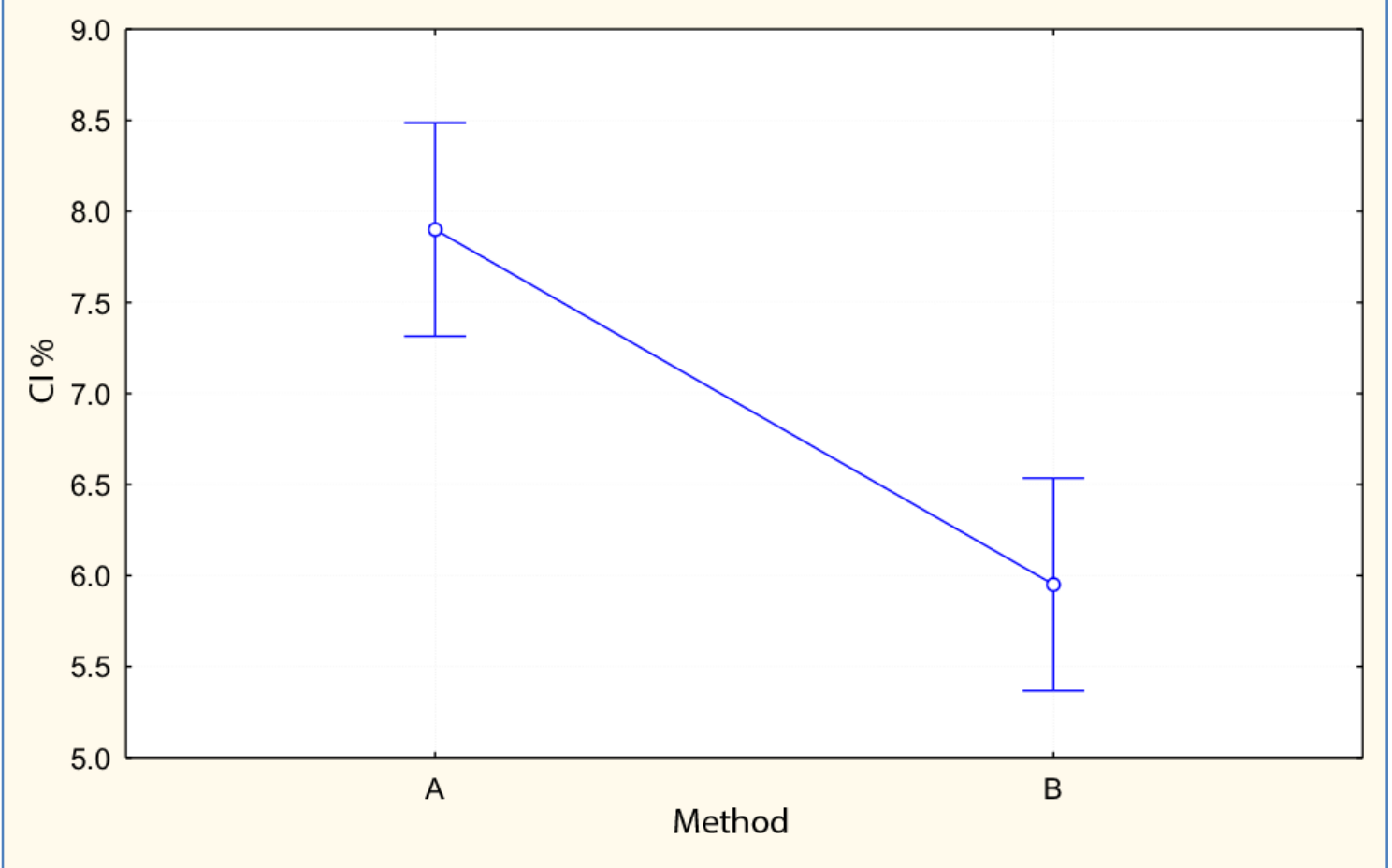

Figure 1: Comparison between mean Cl\% obtained with Method $\mathrm{A}$ to the one obtained with Method B: vertical segments show confidence interval (95\%).

Secondly the samples tested were grouped according to the three variables Category, Filter and Texture indicated in Table I. Tables V, VI and VII summarize the results for each method, following the grouping.

\begin{tabular}{|l|c|c|c|c|}
\hline \multicolumn{5}{|c|}{ SPF Category } \\
& $\begin{array}{c}\text { Low } \\
\text { (7) }\end{array}$ & $\begin{array}{c}\text { Medium } \\
(16)\end{array}$ & $\begin{array}{c}\text { High } \\
(29)\end{array}$ & Very High \\
& $8,42 \%$ & $6,53 \%$ & $8,80 \%$ & $8,06 \%$ \\
\hline Method A, 100g & $9,37 \%$ & $8,86 \%$ & $7,45 \%$ & $6,90 \%$ \\
\hline Method A, 200g & $6,11 \%$ & $5,93 \%$ & $6,20 \%$ & $6,67 \%$ \\
\hline Method B, 100g & $6,21 \%$ & $5,66 \%$ & $5,89 \%$ & $5,08 \%$ \\
\hline Method B, 200g & & &
\end{tabular}

Table V: Distribution of the tested products by SPF category and corresponding mean $\mathrm{Cl} \%$ for each method.

This article is protected by copyright. All rights reserved. 


\begin{tabular}{|l|c|c|c|}
\hline \multicolumn{3}{|c|}{ Filter } \\
\hline & $\begin{array}{c}\text { Chemical C } \\
(23)\end{array}$ & $\begin{array}{c}\text { Physical P } \\
(9)\end{array}$ & $\begin{array}{c}\text { Combination CP } \\
(43)\end{array}$ \\
\hline Method A, 100g & $7,88 \%$ & $8,59 \%$ & $7,95 \%$ \\
\hline Method A, 200g & $8,87 \%$ & $8,84 \%$ & $7,10 \%$ \\
\hline Method B, 100g & $6,80 \%$ & $7,02 \%$ & $5,85 \%$ \\
\hline Method B, 200g & $6,08 \%$ & $5,78 \%$ & $5,36 \%$ \\
\hline
\end{tabular}

Table VI: Distribution of the tested products by type of filters and corresponding mean $\mathrm{Cl} \%$ for each method.

\begin{tabular}{|l|c|c|c|c|}
\hline \multicolumn{5}{|c|}{ Texture } \\
\hline & $\begin{array}{c}\text { Liquid } \\
(10)\end{array}$ & $\begin{array}{c}\text { Fluid } \\
(38)\end{array}$ & $\begin{array}{c}\text { Creamy } \\
(18)\end{array}$ & $\begin{array}{c}\text { Paste } \\
(9)\end{array}$ \\
\hline Method A, 100g & $8,65 \%$ & $7,60 \%$ & $8,18 \%$ & $8,70 \%$ \\
\hline Method A, 200g & $8,35 \%$ & $7,88 \%$ & $6,81 \%$ & $8,75 \%$ \\
\hline Method B, 100g & $6,42 \%$ & $6,31 \%$ & $5,98 \%$ & $6,47 \%$ \\
\hline Method B, 200g & $7,78 \%$ & $5,38 \%$ & $4,94 \%$ & $5,66 \%$ \\
\hline
\end{tabular}

Table VII: Distribution of the tested products by texture and corresponding mean $\mathrm{Cl} \%$ for each method.

Leaving aside the pressure and only considering the method as significant variable, we studied the statistical relation between the method and SPF category, filter and texture respectively, according to the mean $\mathrm{Cl} \%$ of the analysis (Table VIII, IX and X). We can conclude that also in this case the choice of the method is found to be the only significant variable able to affect the repeatability of the measure.

\begin{tabular}{|l|c|c|c|c|c|}
\hline & SS & df & MS & F & $p$ \\
\hline Intercept & 10147.49 & 1 & 10147.49 & 792.2758 & 0.000000 \\
\hline Method & 198.10 & 1 & 198.10 & 15.4666 & $\mathbf{0 . 0 0 0 1 0 6}$ \\
\hline Filter & 52.13 & 2 & 26.07 & 2.0352 & $\mathbf{0 . 1 3 2 5 4 8}$ \\
\hline Method*Filter & 0.32 & 2 & 0.16 & 0.0125 & $\mathbf{0 . 9 8 7 6 1 0}$ \\
\hline Error & 3650.29 & 285 & 12.81 & & \\
\hline
\end{tabular}

Table VIII: Statistical analysis Mean Cl\% vs Method and Filter: the mean Cl\% values were statistically compared by a two-way analysis of variance (ANOVA), taking Method and Filter as variables.

This article is protected by copyright. All rights reserved. 


\begin{tabular}{|l|c|c|c|c|c|}
\hline & SS & df & MS & F & $p$ \\
\hline Intercept & 10880.58 & 1 & 10880.58 & 845.9816 & 0.000000 \\
\hline Method & 212.98 & 1 & 212.98 & 16.5594 & $\mathbf{0 . 0 0 0 0 6 1}$ \\
\hline Texture & 54.66 & 3 & 18.22 & 1.4167 & $\mathbf{0 . 2 3 8 0 7 9}$ \\
\hline Method*Texture & 7.73 & 3 & 2.58 & 0.2004 & $\mathbf{0 . 8 9 6 0 9 5}$ \\
\hline Error & 3639.80 & 283 & 12.86 & & \\
\hline
\end{tabular}

Table IX: Statistical analysis Mean Cl\% vs Method and Texture: the mean CI\% values were statistically compared by a two-way analysis of variance (ANOVA), taking Method and Texture as variables.

\begin{tabular}{|l|c|c|c|c|c|}
\hline & SS & df & MS & F & $p$ \\
\hline Intercept & 11014.85 & 1 & 11014.85 & 848.3435 & 0.000000 \\
\hline Method & 239.50 & 1 & 239.50 & 18.4460 & $\mathbf{0 . 0 0 0 0 2 4}$ \\
\hline SPF Category & 20.45 & 3 & 6.82 & 0.5251 & $\mathbf{0 . 6 6 5 3 5 5}$ \\
\hline Method*SPF Category & 7.92 & 3 & 2.64 & 0.2033 & $\mathbf{0 . 8 9 4 0 3 6}$ \\
\hline Error & 3674.46 & 283 & 12.98 & & \\
\hline
\end{tabular}

Table X: Statistical analysis Mean Cl\% vs Method and SPF Category: the mean $\mathrm{Cl} \%$ values were statistically compared by a two-way analysis of variance (ANOVA), taking Method and SPF Category as variables.

An analogue analysis was performed considering pressure in relation to three variable (SPF category, filter and texture) and, as expected; no statistically significant combination of parameters was highlighted (statistical data not showed).

\section{Correlation between in vitro and in vivo data}

Because the only officially accepted method for SPF determination is the in vivo procedure, we next compared the SPF in vitro results of 11 selected products with their SPF in vivo, to prove the accuracy of the methods, independently from that reported in the label of the products. The results are summarized in Table XI.

This article is protected by copyright. All rights reserved. 


\begin{tabular}{|c|c|c|c|c|c|c|c|c|c|c|}
\hline & \multicolumn{2}{|c|}{ In vivo } & \multicolumn{2}{|c|}{ In vitro $A 1$} & \multicolumn{2}{|c|}{ In vitro $A 2$} & \multicolumn{2}{|c|}{ In vitro $B 1$} & \multicolumn{2}{|c|}{ In vitro $B 2$} \\
\hline rod. & SPF & $\mathrm{Cl} \%$ & SPF & $\mathrm{Cl} \%$ & SPF & $\mathrm{Cl} \%$ & SPF & $\mathrm{Cl} \%$ & $S P F$ & $\mathrm{Cl} \%$ \\
\hline 1 & 6,1 & 15,6 & 9,63 & 12,63 & 12,79 & 10,55 & 6,58 & 8,98 & 5,19 & 10,30 \\
\hline 2 & 6,9 & J, & 1,71 & 6,88 &, 52 & 6,83 & 9,28 & 2,24 & 8,9 & 2,20 \\
\hline 15 & 18,8 & 9 &, 88 & 3,27 & 25,88 & 5,36 & 35,5 & 5,60 & 26,4 & 3,24 \\
\hline 20 & 20,3 & 7,5 & 38,16 & 10,37 & 42,74 & 6,86 & 38,68 & 8,44 & 31,54 & 4,10 \\
\hline 35 & 5,1 & 12,5 & 5,38 & 9,72 & 5,85 & & 2,04 & 1,76 & 2,03 & 1,72 \\
\hline 36 & 30,6 & e & 97 & 5,76 & 39,02 & 6 & 1,76 & 13,71 & 30,03 & 7,41 \\
\hline 43 & 1,2 & 4,6 & 51,92 & 12,61 & 55,67 & 1,69 & 2,66 & 7,92 & 47,67 & 3,22 \\
\hline 49 & 52 & 7,6 & 41,7 & 7,44 & 51,08 & 9,87 & 48,02 & 6,88 & 48,41 & 4,08 \\
\hline 59 & 62,8 & 11,5 & 52,81 & 6,26 & 55,97 & 7,23 & 47,9 & 7,43 & 56,45 & 3,86 \\
\hline 65 & 56,1 & 10,5 & 51,28 & 4,39 & 45,78 & 3,81 & 36,03 & 4,58 & 35,31 & 4,47 \\
\hline 75 & 61,2 & 5,1 & 39,13 & 10,78 & 42,98 & 8,98 & 17,21 & 2,91 & 28,94 & 14,48 \\
\hline
\end{tabular}

Table XI: SPF results of 11 selected product from in vivo and in vitro methods.

To evaluate a possible linear correlation of in vitro and in vivo data, we calculated the Pearson index, reported in Table XII.

\begin{tabular}{|l|c|c|c|c|c|c|c|}
\hline & Mean & Dv.Std. & $r(X, Y)$ & $r^{2}$ & $t$ & $p$ & $N$ \\
\hline vivo & 35.55455 & 20.85969 & & & & & \\
\hline A1 & 34.41545 & 15.32343 & 0.849327 & $\mathbf{0 . 7 2 1 3 5 6}$ & 4.826934 & $\mathbf{0 . 0 0 0 9 3 8}$ & 11 \\
\hline vivo & 35.55455 & 20.85969 & & & & & \\
\hline A2 & 38.38909 & 15.15401 & 0.842200 & $\mathbf{0 . 7 0 9 3 0 1}$ & 4.686132 & $\mathbf{0 . 0 0 1 1 4 2}$ & 11 \\
\hline vivo & 35.55455 & 20.85969 & & & & & \\
\hline B1 & 30.51455 & 16.62740 & 0.509246 & $\mathbf{0 . 2 5 9 3 3 2}$ & 1.775159 & $\mathbf{0 . 1 0 9 6 1 1}$ & 11 \\
\hline vivo & 35.55455 & 20.85969 & & & & & \\
\hline B2 & 30.07909 & 16.67036 & 0.743459 & $\mathbf{0 . 5 5 2 7 3 1}$ & 3.334982 & $\mathbf{0 . 0 0 8 7 3 0}$ & 11 \\
\hline
\end{tabular}

Table XII: Pearson correlation of in vitro and in vivo results.

Surprisingly, method A gives best statistically correlated results between in vivo and in vitro data. From these results it can be pointed out that in vitro SPF obtained with Method A significantly correlates with in vivo result for both the pressures applied, being in such way a measure independent from pressure exerted by the operator. The same cannot be confirmed for Method B, where only the data set obtained applying $200 \mathrm{~g}$ pressure (Method B2) is statistically correlated with the in vivo data. Furthermore, it should be observed that, regardless the previous data presented on repeatability, the Methods A do indeed better correlate to in vivo SPF.

This article is protected by copyright. All rights reserved. 




Figure 2: In vitro-in vivo correlation.

\section{Conclusion}

Repeatability and in vivo correlation of in vitro SPF (accuracy) measurements, together with reproducibility, are a puzzling issue already discussed by other authors (24). Furthermore, even if a method appears to be statistically valid and interesting, we must consider that the SPF analysis is not a merely academic exercise, but mostly a manufacturer's must. In this regard, a method must be not only statistically significant, but also predictive and easy to apply to a routine complex development process that deals with ever-complex formulations.

Starting from conflicting results, observed during our routine measurements, the purpose of this study was to evaluate if a standard technique of spreading could statistically improve the results in terms of repeatability and if the SPF calculated could be predictive of the in vivo measurement, which is the standard method for SPF determination of sunscreen products. As stated in the introduction our concern was to elaborate a simple method, applicable not just in scientific investigations, but also by manufacturers with limited equipment.

Data collected and analyzed showed that the standardization of the pressure applied to spread the product on the substrate does not lead to significant improvements of the data variability.

This article is protected by copyright. All rights reserved. 
As confirmed by other authors, the choice of the substrate is much more critical and PMMA plates gives, in terms of repeatability, better results than the "old fashioned" Transpore ${ }^{\mathrm{TM}}$ Tape, although it also has shown acceptable values of $\mathrm{Cl} \%$, with results well below the $17 \%$ threshold.

As is known, the PMMA plates provide more repeatable data, being a more standardized substrate in terms of composition and surface roughness, while the main limit of the Transpore ${ }^{\mathrm{TM}}$ tape is precisely the impossibility to obtain a support with unique characteristics: surface can vary greatly from one batch to another. In this case it is necessary to rely on the experience and know-how of the laboratory to make small adaptations of the protocol in order to correct the differences due to the substrate and this makes impossible the definition of a standard method valid and applicable in any laboratory.

Other variables as SPF category, filters or texture, resulted not statistically significant for the determination of in vitro SPF and therefore it was not possible to identify a better method according to these subsets of products.

Meanwhile, the results obtained from the linear correlation of in vitro SPF and the in vivo data obtained applying ISO 24444 were completely unexpected: for the 11 products selected the best correlation could be obtained with Method A, using the Transpore ${ }^{\mathrm{TM}}$ Tape and, moreover, the correlation was not influenced by the operator's pressure.

A significant correlation with in vivo test was found for Method B (using PMMA plates) just for the $200 \mathrm{~g}$ pressure of application.

Based on the results of our investigation, we can conclude that the Method B2 is the most reliable according to the data repeatability and accuracy. Nevertheless, the method A with Transpore ${ }^{\mathrm{TM}}$ tape can still be considered an in vitro method predictive of the in vivo SPF during the research and development phases of the solar product, especially in laboratories with limited financial resources and limited equipment.

Further work is currently ongoing to compare influence of different kind of formulations, frequently used by product developers, in order to devise possible modifications to the methods proposed, to obtain better correlation to in vivo test results.

The present study was conducted entirely in our laboratory, in highly standardized operating conditions with regard to the operator, environmental conditions, the substrates used and the instruments, in order to evaluate whether the application pressure of the sample on the substrate and the intrinsic characteristics of the formulations could affect the result. As the considered variables did not provide significantly relevant results, it may be assumed that the difficulty in defining a single protocol for the determination of sunscreen in vitro SPF could be due to "external variables" (eg. environmental, operator), which are more difficult to control in different laboratories.

This article is protected by copyright. All rights reserved. 
This also explains the evidence that it is possible for a single laboratory to optimize internal methods and protocols to achieve repeatable and predictive in vitro results, as we demonstrated in this work, while it is extremely difficult to make methods reproducible and equally reliable in different laboratories.

For these reasons, as concluded by other authors (25), we support the need of a common room for discussion, to compare methods between different laboratories in order to devise a common protocol, which takes into consideration the environmental variables.

\section{-Acknowledgements}

S.M. and S.V. thank Ministry of Education, University and Research (MIUR Italy- PRIN, Grant 20105YY2HL_006) and Ambrosialab Srl (Ferrara) for the financial support.

1. Miksa, S., Lutz, D., Guy, C. New approach for a reliable in vitro sun protection factor method Part I: Principle and mathematical aspects. Vol. 37, International Journal of Cosmetic Science. 2015. p. 555-66.

2. European Commission. Regulation (EC) No $1223 / 2009$ of the European Parliament and of the Council of 30 November 2009 on cosmetic products. Off J Eur Union. 2009;(1223):342-59.

3. Commission Recommendation of 22 September 2006 on the efficacy of sunscreen products and the claims made relating thereto (2006/647/EC). Off J Eur Union.

4. ISO. The Standard EN ISO 24443:2012 Cosmetics - Sun protection test methods - In vitro determination of sunscreen UVA photoprotection. 2012;

5. ISO. EN ISO 24444:2010 Cosmetics - Sun protection test methods. In vivo determination of the sun protection factor (SPF). 2010;

6. Miksa, S., Lutz, D., Guy, C. In vitro/vivo SPF Correlation and Repeatability According to Substrate. Cosmet Toilet. 2013;128(9):648.

7. Sheu, M.T., Lin, C.W., Huang, M.C., Shen, C.H., Ho, H.O. Correlation of in vivo and in vitro measurements of sun protection factor. J Food Drug Anal. 2003;11(2):128-32.

8. Pelizzo, M., Zattra, E., Nicolosi, P., Peserico, A., Garoli, D., Alaibac, M. In vitro evaluation of sunscreens: an update for the clinicians. ISRN Dermatol. 2012;2012(August 2007):352135.

9. Diffey, B.L., Robson, J. A new substrate to measure sunscreen protection factors throughout the ultraviolet spectrum. J Soc Cosmet Chem. 1989;40(June):127-33.

10. Garoli, D., Pelizzo, M.G., Nicolosi, P., Peserico, A., Tonin, E., Alaibac, M. Effectiveness of different substrate materials for in vitro sunscreen tests. J Dermatol Sci. 2009;56(2):89-98.

This article is protected by copyright. All rights reserved. 
11. Dutra, E.A., Almança, D., Kedor, E.R.M., Inês, M., Miritello, R. Determination of sun protection factor ( SPF ) of sunscreens by ultraviolet spectrophotometry. Brazilian J Pharm Sci. 2004;40(3):381-5.

12. Garoli, D., Pelizzo, M.G., Bernardini, B., Nicolosi, P., Alaibac, M. Sunscreen tests: Correspondence between in vitro data and values reported by the manufacturers. J Dermatol Sci. 2008;52(3):193-204.

13. Fageon, L., Moyal, D., Coutet, J., Candau, D. Importance of sunscreen products spreading protocol and substrate roughness for in vitro sun protection factor assessment. Int $\mathrm{J}$ Cosmet Sci. 2009;31(6):405-17.

14. Heinrich, U., Tronnier, H., Kockott, D., Kuckuk, R., Heise, H.M. Comparison of sun protection factors determined by an in vivo and different in vitro methodologies: A study with 58 different commercially available sunscreen products. Int J Cosmet Sci. 2004;26(2):79-89.

15. Couteau, C., Paparis, E., Coiffard, L.J.M. Sun oils: effect of the applied dose on SPF determined by using in vitro method. Pharm Dev Technol. 2014;19(3):315-7.

16. Fujikake, K., Tago, S., Plasson, R., Nakazawa, R., Okano, K., Maezawa, D. et al. Problems of in vitro SPF measurements brought about by viscous fingering generated during sunscreen applications. Skin Pharmacol Physiol. 2014;27(5):254-62.

17. Cariou, N. Sunscreen in-vitro SPF determination - inter and intra comparison tests between several measurement instruments. 2012;22-4.

18. Pissavini, M., Marguerie, S., Dehais, A., Ferrero, L., Zastrow, L. Characterizing Roughness: A New Substrate to Measure SPF. Cosmet Toilet. 2009;124(9):56-64.

19. Sohn, M., Hêche, A., Herzog, B., Imanidis, G. Film thickness frequency distribution of different vehicles determines sunscreen efficacy. J Biomed Opt. 2014;19(11):1-11.

20. Moyal, D., Alard, V., Bertin, C., Boyer, F., Brown, M.W., Kolbe, L. et al. The revised COLIPA in vitro UVA method. Int J Cosmet Sci. 2013;35(1):35-40.

21. Miksa, S., Lutz, D., Guy, C., Labs, H. Influence of Pressure During Spreading on UV Transmission Results Media. Cosmet Toilet. 2013;128(11):822-35.

22. COLIPA TECT and PA-. International sun protection factor (SPF) test method. Int Sun Prot Factor Test Method. 2006;(May).

23. Ferrero, L., Pissavini, M., Dehais, A., Marguerie, S., Zastrow, L., et al. Importance of Substrate Roughness for In Vitro Sun Protection In Vitro Sun Protection Assessment. IFSCC Mag. 2006;9(2).

This article is protected by copyright. All rights reserved. 
24. Rohr, M., Klette, E., Ruppert, S., Bimzcok, R., Klebon, B., Heinrich, U. et al. In vitro Sun Protection Factor: Still a Challenge with No Final Answer. Skin Pharmacol Physiol. 2010;23(4):201-12.

25. Batzer, J., Bleckmann, A., Lerg, H., Schwanke, F., Schläger, T. The "Dispersal Rate" - a product dependent characteristic to predict the reliability of the calibrated in vitro SPF on WW5 plates. Int J Cosmet Sci. 2016;38(3):294-304.

This article is protected by copyright. All rights reserved. 